\title{
Extended gradient plasticity by the subloading surface model with tangential plasticity
}

\author{
Koichi HASHIGUCHI*, Mehdi KHOJASTEHPOUR** and Saichi SAKAJO*** \\ * Dr. Eng and Dr. Agr., Professor, Dept. Prod. Environment. Sci., Kyushu University, \\ Hakozaki, Higashi-ku, Fukuoka 812-8581 \\ ** M. Agr., Dept. Prod. Environment. Sci., Kyushu University, \\ Hakozaki, Higashi-ku, Fukuoka 812-8581 \\ *** Dr. Eng., Appl. Inform. Sci. Dept., Kiso-jiban Consultants Co. Ltd., \\ Kudan-kita 1-11-5, Chiyoda-ku, Tokyo 102-8220
}

\begin{abstract}
The most useful non-local constitutive equation for the analysis of localized plastic deformation would be the gradient plastic constitutive equation proposed by Aifantis ${ }^{1), 2)}$. However, it involves the gradient of internal variable and thus the treatment of it at the boundary is difficult. Then, it has been modified mathematically by Vardoulakis ${ }^{3)-5)}$ transforming it to the relationship between the stress rate and the strain rate with its gradient, and thus the analysis of the boundary value problem has been simplified drastically. In this article the generalized formulation of the gradient plasticity is first given by incorporating the general gradient terms of internal variables. Then, it is extended to the unconventional plasticity ${ }^{6}$ by incorporating the subloading surface model ${ }^{7)-10)}$. Moreover, it is extended so as to be applicable to the description of plastic instability phenomena by incorporating the tangential plastic strain rate ${ }^{11)-13}$. Besides, the equation for the analysis of shear band thickness in the post-localized deformation is given based on the present constitutive model.

Key Words: elastoplasticity, gradient theory, subloading surface model, tangential plasticity
\end{abstract}

\section{Introduction}

A non-local constitutive equation involving a characteristic length has to be adopted in order to predict the localized deformation appropriately. The most useful non-local constitutive equation for the analysis of plastic deformation inducing a localization seems to be the gradient plasticity advocated by Aifantis ${ }^{1), 2)}$ extending the yield condition so as to depend not only on the internal variable but also on its gradient in order to take the state of internal structure in the surrounding region into account. However, the constitutive equation involves the gradient of internal variable itself and thus this fact leads to the difficulty in its treatment at the boundary of materials. Then, this approach was modified mathematically by Vardoulakis ${ }^{3)-5)}$ transforming the constitutive equation to the relationship between the stress rate and the strain rate with its gradient, and thus the analysis of the boundary value problem has been simplified drastically. However, it involves only the second-order gradient term of a single isotropic hardening variable is incorporated. Further, it falls within the conventional plasticity ${ }^{6}$ premising that the interior of the yield surface is a purely elastic domain and thus it cannot predict a plastic deformation due to the change of stress inside the yield surface. In addition, it cannot predict the inelastic strain rate due to the stress rate component tangential to the yield surface, which is of importance in the analysis of plastic instability phenomena. 
In this article the generalized formulation of the gradient plasticity in the Vardoulakis ${ }^{33)-5}$ form is first given by incorporating the general gradient terms of internal variables. Then, it is extended to the unconventional plasticity by incorporating the subloading surface model ${ }^{7)-10)}$. Further, it is extended so as to be applicable to plastic instability phenomena by incorporating the tangential plastic strain rate induced by the stress rate component tangential to the subloading surface $^{11)-13)}$. Besides, the mathematical formulation for the analysis of shear band thickness in post-localized deformation for the plane strain biaxial stretching under a constant volume is given based on the present constitutive model.

\section{Constitutive Equation}

In this section the subloading surface model with tangential stress rate effect will be extended so as to involve the gradient terms.

Denoting the current position vector and the velocity of material particle as $\mathbf{x}$ and $\mathbf{v}$, the velocity gradient is described as $\mathbf{L}=\partial \mathbf{v} / \partial \mathbf{x}$ by which the strain rate and the continuum spin are defined as $\mathbf{D}$ $\left(=\left(\mathbf{L}+\mathbf{L}^{\mathrm{T}}\right) / 2\right)$ and $\mathbf{W}\left(=\left(\mathbf{L}-\mathbf{L}^{\mathrm{T}}\right) / 2\right)$, respectively, ( ) ${ }^{\mathrm{T}}$ standing for the transpose. Let the strain rate $\mathbf{D}$ be additively decomposed into the elastic strain rate $\mathbf{D}^{e}$ and the inelastic strain rate $\mathbf{D}^{i}$, i.e.

$$
\mathbf{D}=\mathbf{D}^{e}+\mathbf{D}^{i},
$$

where $\mathbf{D}^{e}$ is given by

$$
\mathbf{D}^{e}=\mathbf{E}^{-1} \stackrel{\circ}{\boldsymbol{\sigma}} \text {. }
$$

$\boldsymbol{\sigma}$ is the Cauchy stress and $\left(^{\circ}\right)$ indicates the proper corotational rate with the objectivity and the fourth-order tensor $\mathbf{E}$ is the elastic modulus. Further, let the inelastic strain rate $\mathbf{D}^{i}$ be additively decomposed into the plastic strain rate $\mathbf{D}^{p}$ and the tangential strain rate $\mathbf{D}^{t}$, i.e.

$$
\mathbf{D}^{i}=\mathbf{D}^{p}+\mathbf{D}^{t}
$$

provided that $\mathbf{D}^{p}$ and $\mathbf{D}^{t}$ are induced by the stress rate component normal and tangential, respectively, to the yield and/or loading surface.

Let the following yield condition be assumed.

$$
f(\boldsymbol{\sigma}, \mathbf{H})=F(H),
$$

where the scalar $H$ and the second-order tensor $\mathbf{H}$ are the isotropic and the anisotropic hardening variables, respectively. The function $f$ is assumed to be homogeneous of degree one in the stress $\boldsymbol{\sigma}$. Then, if $\mathbf{H}=$ const. , the yield surface keeps a similar shape.

Drucker ${ }^{13)}$ defined the unconventional plasticity as the extended plasticity such that the interior of the yield surface is not a purely elastic domain but a plastic deformation is induced by the rate of stress inside the yield surface. The most appropriate model falling within the framework of the unconventional plasticity would be the subloading surface model ${ }^{7)-10)}$.

In the subloading surface model the conventional yield surface is renamed as the normal-yield surface, since its interior is not regarded as a purely elastic domain. Then, let the subloading surface be introduced, which always passes through the current stress point $\boldsymbol{\sigma}$ and also keeps the shape similar to the normal-yield surface and the orientation of similarity. The similarity and the orientation of similarity of surfaces possess the following geometrical properties.

i ) All lines connecting an arbitrary point on or within the subloading surface and its conjugate point on or within the normal-yield surface join at the specific point called the similarity-center.

ii ) All ratios of the length of an arbitrary line-element connecting two points on or inside the subloading surface and that of an arbitrary conjugate line-element connecting two conjugate points on or inside the normal-yield surface are identical. The ratio is called the similarity-ratio which coincides with the ratio of the sizes of these surfaces.

Let the similarity-ratio of the subloading surface to the normal-yield surface be specifically called the normal-yield ratio, and let it be denoted by $R$, where $R=0$ corresponds to the null stress state, $0<R<1$ to the subyield state, $R=1$ to the normal-yield state in which the stress lies on the normal-yield surface. Therefore, the normal-yield ratio $R$ plays the role of the three-dimensional measure of

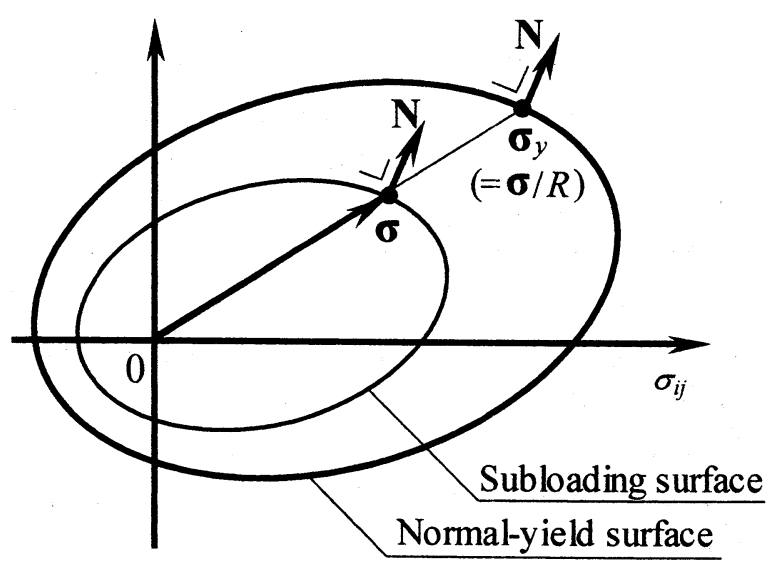

Fig. 1. Normal-yield and subloading surfaces. 
the degree of approach to the normal-yield state.

Then, the subloading surface is described as

$$
f(\boldsymbol{\sigma}, \mathbf{H})=R F(H),
$$

The normal-yield and the subloading surfaces are illustrated in Fig. 1, where $\boldsymbol{\sigma}_{y}(\equiv \boldsymbol{\sigma} / R)$ is the conjugate stress on the normal-yield surface at which the normalized outward-normal $\mathbf{N}$ is same as that at the current stress $\boldsymbol{\sigma}$ on the subloading surface.

Eq. (5) is extended to the non-local subloading surface assuming that the variables evolving by the plastic deformation are non-local depending not only on the current point but also on a certain neighboring region as follows:

$$
f(\boldsymbol{\sigma},\langle\mathbf{H}\rangle)=\langle R F(H)\rangle,
$$

where \langle\rangle designates the averaging value in an appropriate surrounding region. The averaging can be given by

$$
\langle\rangle=\nabla_{g}[]
$$

where

$$
\nabla g \equiv 1+c_{2}^{2} \nabla^{2}+c_{4}^{4} \nabla^{4}+\cdots
$$

$c_{2}, c_{4}, \ldots$ are the material constants depending on the characteristic (internal) length. Then, Eq. (6) can be written as

$$
f(\boldsymbol{\sigma}, \nabla g[\mathbf{H}])=\nabla g[R F(H)] .
$$

The material-time derivative of Eq. (9) is given as

$$
\operatorname{tr}\left\{\frac{\partial f\left(\boldsymbol{\sigma}, \nabla_{g}[\mathbf{H}]\right)_{\circ}}{\partial \boldsymbol{\sigma}}\right\}+\operatorname{tr}\left\{\frac{\partial f\left(\boldsymbol{\sigma}, \nabla_{g}[\mathbf{H}]\right)}{\partial \nabla_{g}[\mathbf{H}]} \nabla_{g}[\stackrel{\circ}{\mathbf{H}}]\right\}
$$

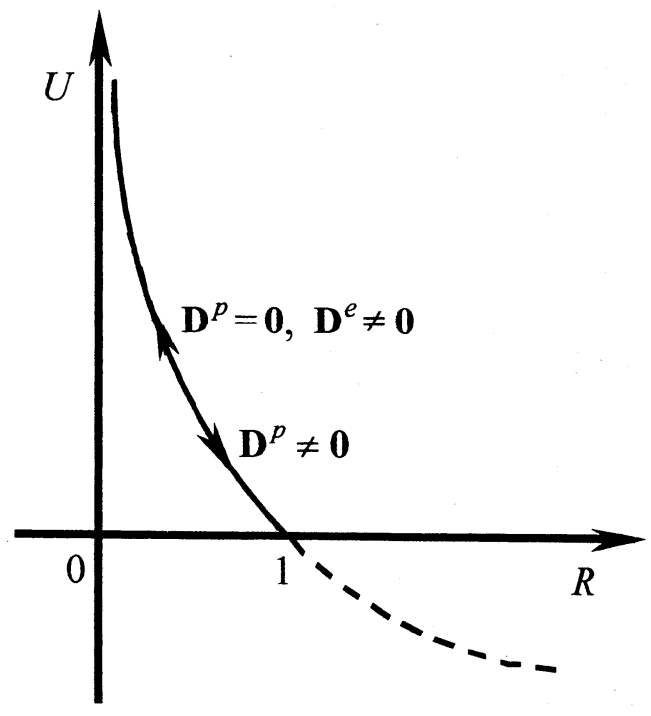

Fig. 2. The function $U$ in the evolution rule of the normal-y ield ratio $R$.

$$
=\nabla g[\dot{R} F+R \dot{F}],
$$

where $\left({ }^{\bullet}\right)$ stands for the material-time derivative.

As observed in experiments, the stress asymptotically approaches the normal-yield surface in the plastic loading process $\mathbf{D}^{p} \neq \mathbf{0}$. Thus, the following evolution equation of the normal-yield ratio $R$ is assumed.

$$
\dot{R}=U\left\|\mathbf{D}^{p}\right\| \text { for } \mathbf{D}^{p} \neq \mathbf{0},
$$

where $U$ is a monotonically decreasing function of the normal-yield ratio $R$, fulfilling (see Fig. 2)

$$
\begin{aligned}
& U= \begin{cases}\infty & \text { for } R=0, \\
0 & \text { for } R=1,\end{cases} \\
& (U<0 \text { for } R>1) .
\end{aligned}
$$

Let the function $U$ satisfying Eq. (12) be simply given by

$$
U=-u \ln R,
$$

where $u$ is the material constant.

The substitution of Eq. (11) into Eq. (10) leads to the consistency condition extended to the subloading surface:

$$
\begin{array}{r}
\operatorname{tr}\left\{\frac{\partial f(\boldsymbol{\sigma}, \nabla g[\mathbf{H}])}{\partial \boldsymbol{\sigma}} \stackrel{\circ}{\boldsymbol{\sigma}}\right\}+\operatorname{tr}\left\{\frac{\partial f(\boldsymbol{\sigma}, \nabla g[\mathbf{H}])}{\partial \nabla_{g}[\mathbf{H}]} \nabla_{g}[\stackrel{\circ}{\mathbf{H}}]\right\} \\
=\nabla_{g}\left[U\left\|\mathbf{D}^{p}\right\| F+R F^{\prime} \stackrel{\bullet}{H}\right],
\end{array}
$$

where

$$
F^{\prime} \equiv \frac{d F}{d H} .
$$

Adopt the associated flow rule

$$
\mathbf{D}^{p}=\lambda \mathbf{N},
$$

where $\lambda$ is the positive proportionality factor and

$$
\mathbf{N} \equiv \frac{\partial f(\boldsymbol{\sigma}, \mathbf{H})}{\partial \boldsymbol{\sigma}} /\left\|\frac{\partial f(\boldsymbol{\sigma}, \mathbf{H})}{\partial \boldsymbol{\sigma}}\right\| \quad(\|\mathbf{N}\|=1)
$$

The substitution of Eq. (16) into Eq. (14) leads to

$$
\begin{aligned}
\operatorname{tr}\left\{\frac{\partial f(\boldsymbol{\sigma}, \nabla g[\mathbf{H}])}{\partial \boldsymbol{\sigma}}\right. & \stackrel{\circ}{\boldsymbol{\sigma}}\}+\operatorname{tr}\left\{\frac{\partial f(\boldsymbol{\sigma}, \nabla g[\mathbf{H}])}{\partial \nabla_{g}[\mathbf{H}]} \nabla_{g}[\lambda \mathbf{h}]\right\} \\
& =\nabla_{g}\left[U \lambda F+R F^{\prime} \lambda h\right]
\end{aligned}
$$

where $h$ and $\mathbf{h}$ are functions of stress, plastic internal state variables and $\mathbf{N}$ of homogeneous degree one, while these functions are related to $\stackrel{\dot{H}}{\text { and }} \stackrel{\circ}{\mathbf{H}}$ as

$$
h \equiv \frac{\stackrel{\circ}{H}}{\lambda}, \quad \mathbf{h} \equiv \frac{\stackrel{\circ}{\mathbf{H}}}{\lambda} .
$$


In the enough approximation Eq. (18) reduces to

$$
\begin{aligned}
\operatorname{tr}\left\{\frac{\partial f(\boldsymbol{\sigma}, \mathbf{H})}{\partial \boldsymbol{\sigma}} \stackrel{\circ}{\boldsymbol{\sigma}}\right\} & +\operatorname{tr}\left\{\frac{\partial f(\boldsymbol{\sigma}, \mathbf{H})}{\partial \mathbf{H}} \mathbf{h} \nabla g[\lambda]\right\} \\
& =\left(U F+R F^{\prime} h\right) \nabla_{g}[\lambda]
\end{aligned}
$$

from which one has

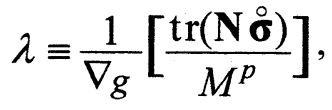

where

$$
M^{p} \equiv\left\{\frac{F^{\prime}}{F} h-\frac{1}{R F} \operatorname{tr}\left(\frac{\partial f(\boldsymbol{\sigma}, \mathbf{H})}{\partial \mathbf{H}} \mathbf{h}\right)+\frac{U}{R}\right\} \operatorname{tr}(\mathbf{N} \boldsymbol{\sigma})
$$

by use of the following relation based on Euler's theorem for a homogeneous function.

$$
\begin{aligned}
\frac{\partial f(\boldsymbol{\sigma}, \mathbf{H})}{\partial \boldsymbol{\sigma}} & =\left\|\frac{\partial f(\boldsymbol{\sigma}, \mathbf{H})}{\partial \boldsymbol{\sigma}}\right\| \mathbf{N}=\frac{\operatorname{tr}\left(\frac{\partial f(\boldsymbol{\sigma}, \mathbf{H})}{\partial \boldsymbol{\sigma}} \boldsymbol{\sigma}\right)}{\operatorname{tr}(\mathbf{N} \boldsymbol{\sigma})} \mathbf{N} \\
& =\frac{f(\boldsymbol{\sigma}, \mathbf{H})}{\operatorname{tr}(\mathbf{N} \boldsymbol{\sigma})} \mathbf{N}=\frac{R F}{\operatorname{tr}(\mathbf{N} \boldsymbol{\sigma})} \mathbf{N} .
\end{aligned}
$$

The positive proportionality factor $\lambda$ in Eq. (21) can be approximated as

$$
\lambda \cong \bar{\nabla} g\left[\frac{\operatorname{tr}(\mathbf{N} \stackrel{\circ}{\mathbf{\sigma}})^{2}}{M^{p}}\right] \cong \frac{\operatorname{tr}(\mathbf{N} \bar{\nabla} g \stackrel{\circ}{\mathbf{\sigma}})}{M^{p}},
$$

where

$$
\bar{\nabla}_{g} \equiv 1-c_{2}^{2} \nabla^{2}-c_{4}^{4} \nabla^{4}-\cdots .
$$

Then, the plastic strain rate is given from Eqs. (16) and (24) as

$$
\mathbf{D}^{p}=\frac{1}{\nabla g}\left[\frac{\operatorname{tr}(\mathbf{N} \stackrel{\circ}{\mathbf{\sigma}})^{\prime}}{M^{p}}\right] \mathbf{N} \cong \frac{\operatorname{tr}\left(\mathbf{N} \bar{\nabla}_{g} \stackrel{\circ}{\boldsymbol{\sigma}}\right)_{M^{p}}}{\mathbf{N}} .
$$

Further, modifying the tangential strain rate proposed by Hashiguchi ${ }^{11)}$ or Hashiguchi and Tsutsumi ${ }^{12}$, the tangential plastic strain rate was given by Hashiguchi $^{13)}$ as follows:

$$
\mathbf{D}^{t}=\frac{1}{T} \mathbf{E}^{-1} \stackrel{\circ}{\boldsymbol{\sigma}}_{t}^{*}
$$

where

$$
\begin{gathered}
\stackrel{\circ}{*}_{t}^{*}=\stackrel{\circ}{\boldsymbol{\sigma}}^{*}-\stackrel{\circ}{\boldsymbol{\sigma}}_{n}^{*}, \quad \stackrel{\circ}{\boldsymbol{\sigma}}_{n}^{*} \equiv \operatorname{tr}\left(\mathbf{n}^{*} \boldsymbol{\sigma}\right) \mathbf{n}^{*}, \\
\mathbf{n}^{*} \equiv\left(\frac{\partial f(\boldsymbol{\sigma})}{\partial \boldsymbol{\sigma}}\right)^{*} /\left\|\left(\frac{\partial f(\boldsymbol{\sigma})}{\partial \boldsymbol{\sigma}}\right)^{*}\right\|=\frac{\mathbf{N}^{*}}{\left\|\mathbf{N}^{*}\right\|}\left(\left\|\mathbf{n}^{*}\right\|=1\right)
\end{gathered}
$$

$$
T=\frac{\xi}{R^{a}} .
$$

$a$ is a material constant and $\xi$ is a material function of

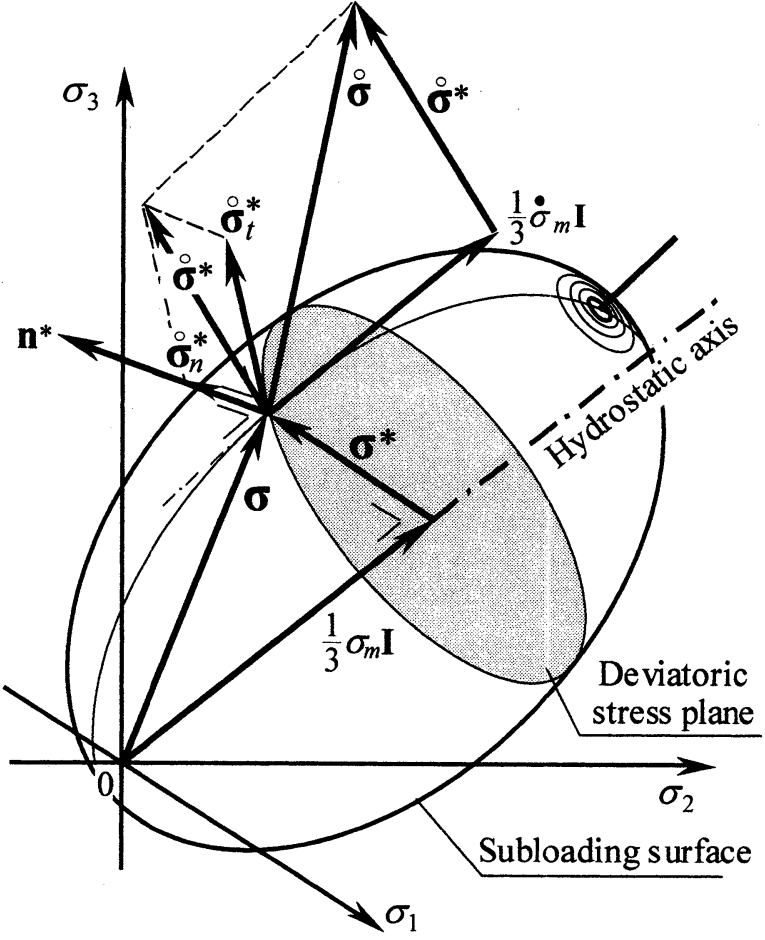

Fig. 3. The deviatoric tangential stress rate $\stackrel{\circ}{\sigma}_{t}^{*}$ illustrated in the principal stress space.

stress and plastic internal variables in general. ( )* designates a deviatoric component. $\mathbf{n}^{*}$ is the normalized-deviatoric outward-normal tensor of the subloading surface, respectively. The stress rate $\stackrel{\circ}{\sigma}_{t}^{*}$ is called the deviatoric-tangential stress rate fulfilling

$$
\operatorname{tr}\left(\mathbf{N} \stackrel{\circ}{\sigma}_{t}^{*}\right)=0, \operatorname{tr} \stackrel{\circ}{\boldsymbol{\sigma}}_{t}^{*}=0 .
$$

The deviatoric-tangential stress rate $\dot{\sigma}_{t}^{*}$ is directed toward the tangential line of the closed curve formed by the intersection of the subloading surface and the deviatoric stress plane as illustrated in Fig. 3.

The tangential strain rate would not influence on the hardening of materials and thus it is assumed that the tangential strain rate is the local variable independent of the neighboring region. Then, the strain rate is given from Eqs. (1), (2), (3), (26) and (27) as

$$
\begin{aligned}
\mathbf{D} & =\mathbf{E}^{-1} \stackrel{\circ}{\boldsymbol{\sigma}}+\frac{1}{\nabla_{g}}\left[\frac{\operatorname{tr}(\mathbf{N} \stackrel{\circ}{\mathbf{\sigma}})}{M^{p}}\right] \mathbf{N}+\frac{1}{T} \mathbf{E}^{-1} \stackrel{\circ}{t}_{t}^{*} \\
& \cong \mathbf{E}^{-1} \stackrel{\circ}{\boldsymbol{\sigma}}+\frac{\operatorname{tr}(\mathbf{N} \bar{\nabla} g \stackrel{\circ}{\boldsymbol{\sigma}})}{M^{p}} \mathbf{N}+\frac{1}{T} \mathbf{E}^{-1} \stackrel{\circ}{t}_{t}^{*}
\end{aligned}
$$

From Eq. (32) one has

$\operatorname{tr}(\mathbf{N E D})=\operatorname{tr}(\mathbf{N} \boldsymbol{\sigma})+\operatorname{tr}(\mathbf{N E N}) \frac{1}{\nabla g}\left[\frac{\operatorname{tr}(\mathbf{N} \boldsymbol{\sigma})}{M^{p}}\right]$ 


$$
=\left\{M^{p} \nabla g+\operatorname{tr}(\mathbf{N E N})\right\} \frac{1}{\nabla g}\left[\frac{\operatorname{tr}(\mathbf{N} \mathbf{\sigma})}{M^{p}}\right]
$$

from which one has

$$
\begin{aligned}
& \frac{1}{\nabla g}\left[\frac{\operatorname{tr}(\mathbf{N} \boldsymbol{\sigma})}{M^{p}}\right]=\frac{1}{M^{p} \nabla g+\operatorname{tr}(\mathbf{N E N})}[\operatorname{tr}(\mathbf{N E D})] \\
& =\frac{1}{1+\frac{M^{p}}{M^{p}+\operatorname{tr}(\mathbf{N E N})}\left(c_{2}^{2} \nabla^{2}+c_{4}^{2} \nabla^{4}+\cdots \cdot\right)} \\
& {\left[\frac{\operatorname{tr}(\mathbf{N E D})}{M^{p}+\operatorname{tr}(\mathbf{N E N})}\right]} \\
& \cong\left\{1-\frac{M^{p}}{M^{p}+\operatorname{tr}(\mathbf{N E N})}\left(c_{2}^{2} \nabla^{2}+c_{4}^{2} \nabla^{4}+\cdots \cdot\right)\right\} \\
& {\left[\frac{\operatorname{tr}(\mathbf{N E D})}{M^{p}+\operatorname{tr}(\mathbf{N E N})}\right]}
\end{aligned}
$$

Then, the positive proportionality factor $\lambda$, rewriting it in terms of strain rate as $\Lambda$, is expressed as

$$
\Lambda=\frac{1}{\nabla\urcorner g}\left[\frac{\operatorname{tr}(\mathbf{N E D})}{M^{p}+\operatorname{tr}(\mathbf{N E N})}\right] \cong \frac{\operatorname{tr}(\mathbf{N E} \bar{\nabla}\urcorner \mathbf{D})}{M^{p}+\operatorname{tr}(\mathbf{N E N})},
$$

where

$$
\begin{aligned}
& \left.\begin{array}{l}
\nabla_{v g} \\
\bar{\nabla}_{v g}
\end{array}\right\}=1 \pm \tilde{\nabla}, \\
& \tilde{\nabla} \equiv \vartheta\left(c_{2}^{2} \nabla^{2}+c_{4}^{2} \nabla^{4}+\cdots\right), \\
& \vartheta \equiv \frac{M^{p}}{M^{p}+\operatorname{tr}(\mathbf{N E N})} .
\end{aligned}
$$

The loading criterion is given as follows ${ }^{14), 15)}$ :

$$
\left.\begin{array}{l}
\mathbf{D}^{p} \neq \mathbf{0}: \Lambda>0, \\
\mathbf{D}^{p}=\mathbf{0}: \Lambda \leq 0
\end{array}\right\}
$$

in terms of the strain rate.

It holds from Eqs. (32) and (33) that

$$
\stackrel{\circ}{\mathbf{\sigma}}=\mathbf{E D}-\mathbf{E} \frac{1}{\nabla \vee g}\left[\frac{\operatorname{tr}(\mathbf{N E D})}{M^{p}+\operatorname{tr}(\mathbf{N E N})}\right] \mathbf{N}-\frac{1}{T} \circ_{t}^{*} \cdot
$$

On the other hand, it is obtained from Eqs. (28) and (38) that

$$
\begin{aligned}
\stackrel{\circ}{\boldsymbol{\sigma}}_{t}^{*}=\stackrel{\circ}{\boldsymbol{\sigma}}^{*}-\operatorname{tr}\left[\mathbf{n}^{*}\{\mathbf{E D}\right. \\
\left.\left.-\mathbf{E} \frac{1}{\nabla v g}\left[\frac{\operatorname{tr}(\mathbf{N E D})}{M^{p}+\operatorname{tr}(\mathbf{N E N})}\right] \mathbf{N}\right\}\right] \mathbf{n}^{*} .
\end{aligned}
$$

The substitution of Eq. (39) into Eq. (38) leads to

$$
\stackrel{\circ}{\boldsymbol{\sigma}}=\mathbf{E D}-\mathbf{E} \frac{1}{\nabla \iota g}\left[\frac{\operatorname{tr}(\mathbf{N E D})}{M^{p}+\operatorname{tr}(\mathbf{N E N})}\right] \mathbf{N}
$$

$$
-\frac{1}{T} \stackrel{\circ}{\boldsymbol{\sigma}}+\frac{1}{T} \dot{\sigma}_{m} \mathbf{I}
$$

$+\frac{1}{T} \operatorname{tr}\left[\mathbf{n}^{*}\left\{\mathbf{E D}-\mathbf{E} \frac{1}{\nabla v g}\left[\frac{\operatorname{tr}(\mathbf{N E D})}{M^{p}+\operatorname{tr}(\mathbf{N E N})}\right] \mathbf{N}\right\}\right] \mathbf{n}^{*}$

On the other hand, it holds from Eq. (38) that

$\dot{\sigma}_{m}=\frac{1}{3}\left\{\operatorname{tr}(\mathbf{E D})-\frac{1}{\nabla v g}\left[\frac{\operatorname{tr}(\mathbf{N E D})}{M^{p}+\operatorname{tr}(\mathbf{N E N})}\right] \operatorname{tr}(\mathbf{E N})\right.$

The substitution of Eq. (41) into Eq. (40) leads to

$$
\begin{aligned}
& \stackrel{\circ}{\mathbf{\sigma}}= \frac{1}{1+T}\left\{T \mathbf{E D}+\frac{1}{3} \operatorname{tr}(\mathbf{E D}) \mathbf{I}+\operatorname{tr}\left(\mathbf{n}^{*} \mathbf{E D}\right) \mathbf{n}^{*}\right. \\
&-\frac{1}{\nabla \vee g}\left[\frac{\operatorname{tr}(\mathbf{N E D})}{M^{p}+\operatorname{tr}(\mathbf{N E N})}\right]\left\{T \mathbf{E N}+\frac{1}{3} \operatorname{tr}(\mathbf{E N}) \mathbf{I}\right. \\
&\left.\left.+\operatorname{tr}\left(\mathbf{n}^{*} \mathbf{E N}\right) \mathbf{n}^{*}\right\}\right](42)
\end{aligned}
$$

which is approximated as

$$
\begin{array}{r}
\stackrel{\circ}{\sigma}=\frac{1}{1+T}\left[T \mathbf{E D}+\frac{1}{3} \operatorname{tr}(\mathbf{E D}) \mathbf{I}+\operatorname{tr}\left(\mathbf{n}^{*} \mathbf{E D}\right) \mathbf{n}^{*}\right. \\
-\frac{\operatorname{tr}(\mathbf{N E} \bar{\nabla} \vartheta \mathbf{D})}{M^{p}+\operatorname{tr}(\mathbf{N E N})}\left\{T \mathbf{E N}+\frac{1}{3} \operatorname{tr}(\mathbf{E N}) \mathbf{I}\right. \\
\left.\left.+\operatorname{tr}\left(\mathbf{n}^{*} \mathbf{E N}\right) \mathbf{n}^{*}\right\}\right] .
\end{array}
$$

Eq. (43) can be rewritten as

$$
\stackrel{\circ}{\mathbf{\sigma}}=\tilde{\mathbf{C}}^{e p t} \mathbf{D},
$$

where the fourth-order tensor $\mathbf{C}^{\text {ept }}$ is the elastoplastic-tangential modulus given by

$$
\tilde{\mathbf{C}}^{e p t} \equiv \mathbf{C}^{e p t}+\mathbf{C}^{p t} \tilde{\nabla}
$$

with

$$
\mathbf{C}^{e p t} \equiv \frac{T \mathbf{E}+\frac{1}{3} \mathbf{I} \otimes \tilde{\mathbf{E}}+\mathbf{n}^{*} \otimes\left(\mathbf{n}^{*} \mathbf{E}\right)}{1+T}-\mathbf{C}^{p t}
$$

$$
\mathbf{C}^{p t} \equiv \frac{\left\{T \mathbf{E N}+\frac{1}{3} \operatorname{tr}(\mathbf{E N}) \mathbf{I}+\operatorname{tr}\left(\mathbf{n}^{*} \mathbf{E N}\right) \mathbf{n}^{*}\right\} \otimes(\mathbf{N E})}{(1+T)\left\{M^{p}+\operatorname{tr}(\mathbf{N E N})\right\}}
$$

where $\tilde{E}_{k l} \equiv E_{r r k l}$.

Further, let the Zaremba-Jaumann rate be used for the corotational rate of the stress $\boldsymbol{\sigma}$ and the second-order tensor variable $\mathbf{H}$, i.e.

$$
\left.\begin{array}{l}
\stackrel{\circ}{\boldsymbol{\sigma}}=\dot{\boldsymbol{\sigma}}-\mathbf{W \sigma}+\boldsymbol{\sigma W}, \\
\stackrel{\circ}{\mathbf{H}}=\dot{\mathbf{H}}-\mathbf{W H}+\mathbf{H W}
\end{array}\right\}
$$

The plastic strain rate (26) is obtained by substituting the associated flow rule (16) into the consis- 
tency condition (14) which is obtained by incorporating the evolution rule (11) of the normal-yield ratio $R$ into the time-differentiation (10) of Eq. (6) for the subloading surface with the gradients of internal variables. Then, the plastic loading process develops gradually as the stress approaches the normal-yield surface, exhibiting a smooth elastic-plastic transition. Thus, the subloading surface model fulfills the smoothness condition ${ }^{15)-18)}$ defined as "the stress rate-strain rate relation (or the stiffness tensor) changes continuously for a continuous change of stress state". This can be expressed mathematically as follows:

$$
\lim _{\delta \boldsymbol{\sigma} \rightarrow \mathbf{0}} \frac{\partial \stackrel{\circ}{\boldsymbol{\sigma}}\left(\boldsymbol{\sigma}+\partial \boldsymbol{\sigma}, \mathbf{S}_{i}, \mathbf{D}\right)}{\partial \mathbf{D}}=\frac{\partial \stackrel{\circ}{\boldsymbol{\sigma}}\left(\boldsymbol{\sigma}, \mathbf{S}_{i}, \mathbf{D}\right)}{\partial \mathbf{D}},
$$

where $\mathbf{S}_{i}(i=1,2,3, \cdots, \mathrm{m})$ denotes collectively scalar- or tensor-valued internal state variables which describe the alteration of the mechanical response due to the irreversible deformation. $\delta()$ stands for an infinitesimal variation and the response of the stress rate to the strain rate in the current state of stress and internal variables is designated by $\stackrel{\circ}{\boldsymbol{\sigma}}\left(\boldsymbol{\sigma}, \mathbf{S}_{i}, \mathbf{D}\right)$. Therefore, the subloading surface model has the notable advantages as follows:

1) A smooth response (e.g. a smooth axial stress-axial logarithmic strain relation in the uniaxial loading) for a smooth monotonic loading is predicted by the subloading surface model. On the other hand, a nonsmooth response is predicted by constitutive

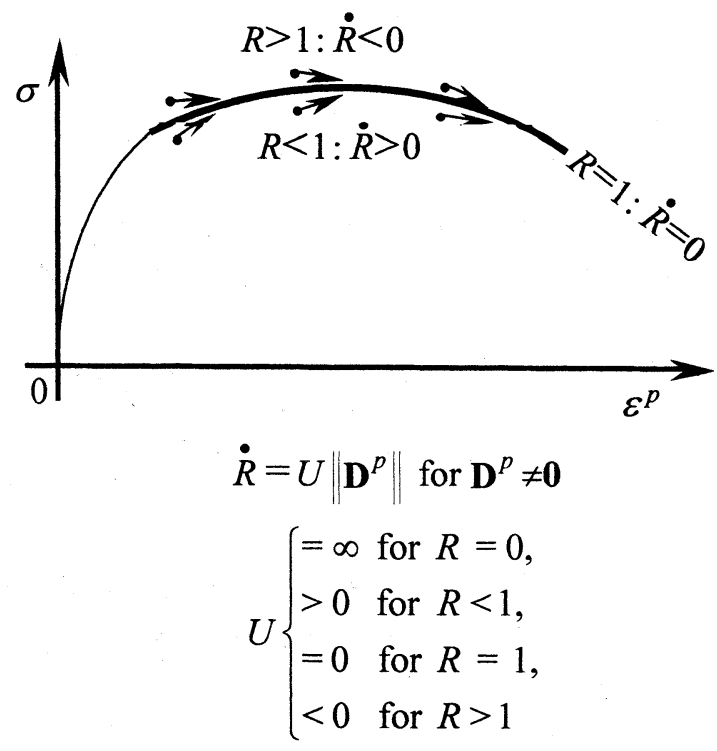

Fig. 4. The stress controlling function contained in the subloading surface model: The stress $\boldsymbol{\sigma}$ is automatically controlled so as to approach the normal-yield surface in the plastic-loading process $\mathbf{D}^{p}$. models violating the smoothness condition as in the conventional plasticity with the yield surface enclosing a purely elastic domain.

2 ) Only the decision for the sign of the proportionality factor $\Lambda$ in terms of strain rate is required in the loading criterion of the subloading surface model, since the stress always lies on the subloading surface, which plays the role of the loading surface, whilst the decision as to whether or not the stress lies on the yield surface is not required. On the other hand, the judgment whether or not the yield condition is fulfilled is required in the conventional plasticity.

3 ) A stress is automatically drawn back to the normal-yield surface even if it goes out from that surface since it is formulated that $\dot{R}>0$ for $R<1$ (subyield state) and $\stackrel{R}{2}<0$ for $R>1$ (over the normal-yield state) in Eq. (11) with the condition (12) (see Fig. 4). Thus, a rough numerical calculation with large loading steps is allowed in the subloading surface model.

Further, the tangential strain rate (27) is also formulated to develop gradually as the stress approaches the normal-yield surface, fulfilling the continuity condition" ${ }^{15)-18)}$ defined as "the stress rate response changes continuously for a continuous change of the strain rate" can be expressed mathematically as follows:

$$
\lim _{\delta \mathbf{D} \rightarrow \mathbf{0}} \stackrel{\circ}{\boldsymbol{\sigma}}\left(\boldsymbol{\sigma}, \mathbf{S}_{i}, \mathbf{D}+\delta \mathbf{D}\right)=\stackrel{\circ}{\boldsymbol{\sigma}}\left(\boldsymbol{\sigma}, \mathbf{S}_{i}, \mathbf{D}\right)
$$

whilst the other tangential plasticity models ${ }^{19)-23)}$ violate the continuity condition predicting an abrupt generation of the tangential strain rate when the stress reaches the yield surface.

\section{Basic Formulation for Post-localization Analysis}

Based on the formulations described in the preceding section, the equations for the prediction of shear band thickness is formulated in this section by extending the approach of Vardoulakis and Aifantis ${ }^{3)}$.

\subsection{Field equations}

The outline of the field equations formulated by Yatomi et al. ${ }^{24)}$ is described below briefly.

At the current time $t$, let the body be bounded by closed surface $a$ and let $\mathbf{t}$ denote the surface traction vector. Then, it is required to satisfy

$$
\int_{a} \mathbf{t} d a=0
$$


for the equilibrium ignoring a body force.

Using the divergence theorem and the relation $\mathbf{t}=\boldsymbol{\sigma n} \quad(\mathbf{n}$ : the outward unit normal to the surface $a$ ), we obtain the equilibrium equation:

$$
\operatorname{div} \boldsymbol{\sigma}=\mathbf{0} .
$$

Noting the basic relation $(d a)^{\bullet}=\mathbf{t}(\operatorname{tr} \mathbf{D}$ $-\mathbf{n} \cdot \mathbf{D n}) d a$, the differentiation of Eq. (52) with respect to time is given by

$$
\int_{a} \underset{\mathbf{t}}{\mathbf{t}} d a=\mathbf{0}
$$

where $\dot{\tilde{\mathbf{t}}}=\dot{\mathbf{t}}+\mathbf{t}(\operatorname{trD}-\mathbf{n} \cdot \mathbf{D n})$ is called the total nominal traction rate. Further, the total nominal (first Piola-Kirchhoff) stress rate $\dot{\mathbf{I}}$ defined by $\stackrel{\boldsymbol{t}}{\mathbf{t}}=\dot{\boldsymbol{\Pi}} \mathbf{n}$ is related to the total Cauchy stress rate $\delta$ by

$$
\dot{\boldsymbol{\Pi}}=\dot{\boldsymbol{\sigma}}+(\operatorname{tr} \mathbf{D}) \boldsymbol{\sigma}-\boldsymbol{\sigma} \mathbf{L}^{\mathrm{T}} \text {. }
$$

Substituting Eq. (48) to Eq. (54), the total nominal stress rate $\dot{\mathbf{I}}$ can be rewritten as

$$
\dot{\mathbf{\Pi}}=\stackrel{\circ}{\sigma}+(\operatorname{trD}) \boldsymbol{\sigma}-\boldsymbol{\sigma D}+\mathbf{W} \boldsymbol{\sigma},
$$

Applying the divergence theorem to Eq. (53), the equilibrium equation is obtained as

$$
\operatorname{div} \dot{\boldsymbol{\Pi}}=\mathbf{0} \text {. }
$$

\subsection{Equations for the analysis of shear band thick-} ness in the plane strain biaxial stretching

Hereinafter, consider the deformation process of the rectangular block subject to continuing homogeneous biaxial loading under the constant volume plane strain condition fulfilling $D_{i i}=0$ and $D_{i 3}=0$ which starts from an isotropic stress state. Here, assume the material isotropy and thus $\sigma_{12}=0$ leading to $N_{12}=n_{12}^{*}=0$ choosing the coordinate system $\left(x_{1}\right.$, $x_{2}$ ) where the axes $x_{1}$ and $x_{2}$ are chosen to the directions of principal stresses $\sigma_{1}$ and $\sigma_{2}$, respectively.

Now, let the elastic modulus tensor $\mathbf{E}$ be given by the Hooke's type as

$$
E_{i j k l}=\left(K-\frac{2}{3} G\right) \delta_{i j} \delta_{k l}+G\left(\delta_{i k} \delta_{j l}+\delta_{i l} \delta_{j k}\right) \text {, }
$$

where $K$ and $G$ are the elastic bulk and shear moduli, respectively, which are functions of the stress and internal state variables in general and $\delta_{i j}$ is the Kronecker's delta, i.e. $\delta_{i j}=1$ for $i=j$ and $\delta_{i j}=0$ for $i \neq j$. It holds for $i, j=1,2$ that

$$
E_{i j k l} N_{k l}=\left\{\begin{array}{l}
\left(K-\frac{2}{3} G\right) N_{r r} \delta_{i j}+2 G N_{i j} \text { for } i=j \\
0 \text { for } i \neq j
\end{array}\right.
$$

$$
\begin{aligned}
& E_{i j k l} D_{k l}=2 G D_{i j}, \\
& N_{i j} E_{i j k l} D_{k l}=2 G\left(N_{11} D_{11}+n_{22} D_{22}\right), \\
& n_{i j}^{*} E_{i j k l} D_{k l}=2 G\left(n_{11}^{*} D_{11}+n_{22}^{*} D_{22}\right) .
\end{aligned}
$$

Then, it holds for Eq. (44) that

$$
\begin{aligned}
\stackrel{\circ}{\sigma}_{11}= & C_{1111}^{e p t} D_{11}+C_{1122}^{e p t} D_{22} \\
& +C_{1111}^{p t} \tilde{\nabla} D_{11}+C_{1122}^{p t} \tilde{\nabla} D_{22}, \\
\stackrel{\circ}{\sigma}_{22}= & C_{2211}^{e p t} D_{11}+C_{2222}^{e p t} D_{22} \\
& +C_{2211}^{p t} \tilde{\nabla} D_{11}+C_{2222}^{p t} \tilde{\nabla} D_{22}, \\
\stackrel{\sigma}{\sigma}_{12} & =2 G D_{12}
\end{aligned}
$$

with which Eq. (55) becomes

$$
\begin{aligned}
\dot{\Pi}_{11}= & C_{1111}^{e p t} D_{11}+C_{1122}^{e p t} D_{22} \\
& +C_{1111}^{p t} \tilde{\nabla} D_{11}+C_{1122}^{p t} \tilde{\nabla} D_{22}-\sigma_{11} D_{11},
\end{aligned}
$$

$\stackrel{\bullet}{\Pi}_{22}=C_{2211}^{e p t} D_{11}+C_{2222}^{e p t} D_{22}$

$$
+C_{2211}^{p t} \tilde{\nabla} D_{11}+C_{2222}^{p t} \tilde{\nabla} D_{22}-\sigma_{22} D_{22},
$$

$$
\dot{\Pi}_{12}=2 G D_{12}-\sigma_{11} D_{12}+W_{12} \sigma_{22} \text {. }
$$

The substitution of Eq. (63) into the equilibrium equation (56) leads to

$$
\begin{aligned}
& C_{111}^{e p t} \partial_{11}^{2} v_{1}+C_{1122}^{e p t} \partial_{21}^{2} v_{2} \\
& +C_{1111}^{p t} \tilde{\nabla} \partial_{11}^{2} v_{1}+C_{1122}^{p t} \tilde{\nabla} \partial_{21}^{2} v_{2} \\
& -\sigma_{11} \partial_{11}^{2} v_{1}+G\left(\partial_{22}^{2} v_{1}+\partial_{12}^{2} v_{2}\right) \\
& -\frac{1}{2} \sigma_{11}\left(\partial_{22}^{2} v_{1}+\partial_{12}^{2} v_{2}\right)+\frac{1}{2} \sigma_{22}\left(\partial_{22}^{2} v_{1}-\partial_{12}^{2} v_{2}\right)=0 \\
& C_{2211}^{e p t} \partial_{12}^{2} v_{1}+C_{2222}^{e p t} \partial_{22}^{2} v_{2} \\
& +C_{2211}^{p t} \tilde{\nabla} \partial_{12}^{2} v_{1}+C_{2222}^{p t} \tilde{\nabla} \partial_{22}^{2} v_{2} \\
& -\sigma_{22} \partial_{22}^{2} v_{2}+G\left(\partial_{21}^{2} v_{1}+\partial_{11}^{2} v_{2}\right) \\
& -\frac{1}{2} \sigma_{11}\left(\partial_{21}^{2} v_{1}+\partial_{11}^{2} v_{2}\right)+\frac{1}{2} \sigma_{22}\left(\partial_{21}^{2} v_{1}-\partial_{11}^{2} v_{2}\right)=0
\end{aligned}
$$

A new coordinate system $(x, y)$ with the axes parallel and perpendicular to the shear band is introduced.

$$
x=x_{1} n_{2}-x_{2} n_{1}, y=x_{1} n_{1}+x_{2} n_{2},
$$

where

$$
n_{1} \equiv-\sin \theta, \quad n_{2} \equiv \cos \theta \text {. }
$$

Noting the relation $\partial / \partial x_{1}=\partial / \partial y \bullet \partial y / \partial x_{1}$, $=n_{1} \partial / \partial y, \partial / \partial x_{2}=\partial / \partial y \bullet \partial y / \partial x_{2}=n_{2} \partial / \partial y, \quad$ Eq. 
(64) reduces to the following system of ordinary differential equations.

$$
\begin{aligned}
& C_{1111}^{e p t} n_{1}^{2} v_{1}^{\prime \prime}+C_{1122}^{e p t} n_{1} n_{2} v_{2}^{\prime \prime} \\
& +C_{1111}^{p t} n_{1}^{2} v_{1}^{\nabla}+C_{1122}^{p t} n_{1} n_{2} v_{2}^{\nabla} \\
& -\sigma_{11} n_{1}^{2} v_{1}^{\prime \prime}+G\left(n_{2}^{2} v_{1}^{\prime \prime}+n_{1} n_{2} v_{2}^{\prime \prime}\right) \\
& \frac{1}{2} \sigma_{11}\left(n_{2}^{2} v_{1}^{\prime \prime}+n_{1} n_{2} v_{2}^{\prime \prime}\right)+\frac{1}{2} \sigma_{22}\left(n_{2}^{2} v_{1}^{\prime \prime}-n_{1} n_{2} v_{2}^{\prime \prime}\right) \\
& C_{2211}^{e p t} n_{1} n_{2} v_{1}^{\prime \prime}+C_{2222}^{e p t} n_{2}^{2} v_{2}^{\prime \prime} \\
& +C_{2211}^{p t} n_{1} n_{2} v_{1}^{\nabla}+C_{2222}^{p t} n_{2}^{2} v_{2}^{\nabla} \\
& -\sigma_{22} n_{2}^{2} v_{2}^{\prime \prime}+G\left(n_{1} n_{2} v_{1}^{\prime \prime}+n_{1}^{2} v_{2}^{\prime \prime}\right) \\
& \quad-\frac{1}{2} \sigma_{11}\left(n_{1} n_{2} v_{1}^{\prime \prime}+n_{1}^{2} v_{2}^{\prime \prime}\right)+\frac{1}{2} \sigma_{22}\left(n_{1} n_{2} v_{1}^{\prime \prime}-n_{1}^{2} v_{2}^{\prime \prime}\right) \\
& \text { where }=0,(67) \\
& \quad()^{\prime \prime} \equiv \frac{\partial^{2}}{\partial y^{2}}, \\
& \quad()^{\nabla} \equiv \vartheta \frac{\partial^{2}}{\partial y^{2}}\left(c_{2}^{2} \frac{\partial^{2}}{\partial y^{2}}+c_{4}^{4} \frac{\partial^{4}}{\partial y^{4}}+\bullet \bullet\right) . \quad \text { (68) }
\end{aligned}
$$$$
=0,(67)
$$

We search for periodic solutions of the system of Eq. (67), which have the form

$$
v_{i}=-\zeta_{i} \sin (Q y)(i=1,2)
$$

and fulfill the following boundary conditions

$$
v_{i}= \pm \zeta_{i} \text { at } y=\mp d_{b} \text { with } v_{i}=0 \text { at } y=0 \text {, }
$$

where $2 d_{b}$ is the shear band thickness. Thus, the wave number $Q$ in Eq. (69) is inversely proportional to the shear-band thickness, i.e.

$$
Q=\frac{\pi}{2 d_{b}} \text {. }
$$

Further, the system of ordinary differential equation (67) reduces to the following algebraic system of equations.

$$
\begin{aligned}
& C_{1111}^{e p t} \alpha^{2} \zeta_{1}+C_{1122}^{e p t} \alpha \zeta_{2} \\
& \quad+C_{1111}^{p t} \alpha^{2} \tilde{Q} \zeta_{1}+C_{1122}^{p t} \alpha \tilde{Q} \zeta_{2} \\
& \quad-\sigma_{11} \alpha^{2} \zeta_{1}+G\left(\zeta_{1}+\alpha \zeta_{2}\right) \\
& \quad-\frac{1}{2} \sigma_{11}\left(\zeta_{1}+\alpha \zeta_{2}\right)+\frac{1}{2} \sigma_{22}\left(\zeta_{1}-\alpha \zeta_{2}\right)=0 \\
& C_{2211}^{e p t} \alpha \zeta+C_{2222}^{e p t} \zeta_{2} \\
& \quad+C_{2211}^{p t} \alpha \tilde{Q} \zeta_{1}+C_{2222}^{p t} \tilde{Q} \zeta_{2} \\
& \quad-\sigma_{22} \zeta_{2}+G\left(\alpha \zeta_{1}+\alpha^{2} \zeta_{2}\right) \\
& \quad-\frac{1}{2} \sigma_{11}\left(\alpha \zeta_{1}+\alpha^{2} \zeta_{2}\right)+\frac{1}{2} \sigma_{22}\left(\alpha \zeta_{1}-\alpha^{2} \zeta_{2}\right)=0
\end{aligned}
$$

where

$$
\begin{gathered}
\alpha \equiv \frac{n_{1}}{n_{2}} . \\
\tilde{Q} \equiv \vartheta\left(-c_{2}^{2} Q^{2}+c_{4}^{4} Q^{4}-c_{6}^{6} Q^{6}+c_{8}^{8} Q^{8} \bullet \bullet\right)
\end{gathered}
$$

Eq. (72) is expressed in the matrix form:

$$
\left[\begin{array}{cc}
C_{111}^{p t} \alpha^{2} \tilde{Q}_{+} C_{111}^{e p t} \alpha^{2} & C_{1122}^{p t} \alpha \tilde{Q}_{+} C_{1122}^{e p t} \alpha \\
-\sigma_{11} \alpha^{2}+G & +G \alpha \\
-\frac{1}{2} \sigma_{11}+\frac{1}{2} \sigma_{22} & -\frac{1}{2} \sigma_{11} \alpha-\frac{1}{2} \sigma_{22} \alpha \\
C_{2211}^{p t} \alpha \tilde{Q}+C_{2211}^{e p t} \alpha & C_{2222}^{p t} \tilde{Q}+C_{2222}^{e p t} \\
+G \alpha & -\sigma_{22}+G \alpha^{2} \\
-\frac{1}{2} \sigma_{11} \alpha+\frac{1}{2} \sigma_{22} & -\frac{1}{2} \sigma_{11} \alpha^{2}-\frac{1}{2} \sigma_{22} \alpha^{2}
\end{array}\right]\left[\begin{array}{l}
\zeta_{1} \\
\zeta_{2}
\end{array}\right]=\left[\begin{array}{l}
0 \\
0 \\
0
\end{array}\right]
$$

The following equation has to hold in order that non-zero solution for $\zeta_{1}$ and $\zeta_{2}$ exists in Eq. (75).

$$
\begin{aligned}
& \left(C_{1111}^{p t} \alpha^{2} \tilde{Q}_{+} C_{1111}^{e p t} \alpha^{2}-\sigma_{11} \alpha^{2}+G-\frac{1}{2} \sigma_{11}+\frac{1}{2} \sigma_{22}\right) \\
& \left(C_{2222}^{p t} \tilde{Q}_{+}+C_{2222}^{e p t}-\sigma_{22}+G \alpha^{2}-\frac{1}{2} \sigma_{11} \alpha^{2}-\frac{1}{2} \sigma_{22} \alpha^{2}\right) \\
& -\left(C_{1122}^{p t} \alpha \tilde{Q}+C_{1122}^{e p t} \alpha+G \alpha-\frac{1}{2} \sigma_{11} \alpha-\frac{1}{2} \sigma_{22} \alpha\right) \\
& \left(C_{2211}^{p t} \alpha \tilde{Q}+C_{2211}^{e p t} \alpha+G \alpha-\frac{1}{2} \sigma_{11} \alpha+\frac{1}{2} \sigma_{22} \alpha\right)=0
\end{aligned}
$$

Noting

$$
\begin{aligned}
C_{1111}^{p t}= & \frac{M^{p}}{(1+T)\left\{M^{p}+\operatorname{tr}(\mathbf{N E N})\right\}^{2}} \\
& \left\{T E_{11 r s} N_{r s}+\operatorname{tr}(\mathbf{n} * \mathbf{E N}) n_{11}^{*}\right\} N_{p q} E_{p q 11}, \\
C_{2222}^{p t} & =\frac{M^{p}}{(1+T)\left\{M^{p}+\operatorname{tr}(\mathbf{N E N})\right\}^{2}} \\
& \left\{T E_{22 r s} N_{r s}+\operatorname{tr}(\mathbf{n} \mathbf{E N}) n_{22}^{*}\right\} N_{p q} E_{p q 22}, \\
C_{1122}^{p t} & =\frac{M^{p}}{(1+T)\left\{M^{p}+\operatorname{tr}(\mathbf{N E N})\right\}^{2}} \\
& \left\{T E_{11 r s} N_{r s}+\operatorname{tr}(\mathbf{n} * \mathbf{E N}) n_{11}^{*}\right\} N_{p q} E_{p q 22}, \\
C_{2211}^{p t} & =\frac{M^{p}}{(1+T)\left\{M^{p}+\operatorname{tr}(\mathbf{N E N})\right\}^{2}} \\
& \left\{T E_{22 r s} N_{r s}+\operatorname{tr}(\mathbf{n} * \mathbf{E N}) n_{22}^{*}\right\} N_{p q} E_{p q 11},
\end{aligned}
$$

and thus

$$
C_{1111}^{p t} C_{2222}^{p t}-C_{1122}^{p t} C_{2211}^{p t}=0 .
$$

Eq. (76) is rewritten as

$$
A_{0}(\alpha) \tilde{Q}+A_{1}(\alpha)=0
$$

where 


$$
\begin{aligned}
A_{0} \equiv & C_{1111}^{p t}\left(C_{2222}^{e p t}-\sigma_{22}+G \alpha^{2}-\sigma^{+} \alpha^{2}\right) \alpha^{2} \\
& +C_{2222}^{p t}\left(C_{1111}^{e p t} \alpha^{2}-\sigma_{11} \alpha^{2}+G-\sigma^{-}\right) \\
& -C_{1122}^{p t}\left(C_{2211}^{e p t}+G-\sigma^{-}\right) \alpha^{2} \\
& -C_{2211}^{p t}\left(C_{1122}^{e p t}+G-\sigma^{+}\right) \alpha^{2}, \\
A_{1} \equiv & \left(C_{1111}^{e p t} \alpha^{2}-\sigma_{11} \alpha^{2}+G-\sigma^{\prime}\right) \\
& \left(C_{2222}^{e p t}-\sigma_{22}+G \alpha^{2}-\sigma^{+} \alpha^{2}\right) \\
- & \left(C_{1122}^{e p t}+G-\sigma^{+}\right)\left(C_{2211}^{e p t}+G-\sigma\right) \alpha^{2},
\end{aligned}
$$

where

$$
\left.\begin{array}{l}
\sigma^{+} \\
\sigma^{-}
\end{array}\right\} \equiv \frac{1}{2}\left(\sigma_{11} \pm \sigma_{22}\right) \text {. }
$$

The orientation of shear band is given by the usual condition of shear band inception

$$
A_{1}=0
$$

as follows:

$$
\left.\begin{array}{c}
\alpha^{2}=\frac{-b \pm \sqrt{b^{2}-4 a c}}{2 a}, \\
a \equiv\left(C_{1111}^{e p t}-\sigma_{11}\right)\left(G-\sigma^{+}\right), \\
b \equiv-\left\{C_{1111}^{e p t} \sigma_{22}+C_{2222}^{e p t} \sigma_{11}\right. \\
\left.+C_{1122}^{e p t}\left(G-\sigma^{-}\right)+C_{2211}^{e p t}\left(G-\sigma^{+}\right)+\sigma_{11} \sigma_{22}\right\} \\
c \equiv\left(C_{2222}^{e p t}-\sigma_{22}\right)\left(G-\sigma^{-}\right)
\end{array}\right\}
$$

For this $\alpha$, the values of $Q$ fulfilling Eq. (78) with Eq. (71) give the shear band thickness $d_{b}$.

\section{Concluding Remarks}

The rational formulation of the gradient theory for the analysis of localized deformation is given and extended to the conventional plasticity incorporating the subloading surface model with the tangential stress rate effect. Besides, the basic formulation for post-localization analysis is given showing the equation for the shear band thickness. Hereinafter, it will be extended further so as to be applicable to the analysis of large deformation by incorporating the large elastoplastic deformation theory of Hashiguchi et al. ${ }^{25}$ ) based on the corotational logarithmic (Hencky) strain theory of Naghdabadi and Saidi ${ }^{26)}$.
Acknowledgements - The author expresses the sincere gratitude to Prof. I. Vardoulakis, Natl. Tech. Univ. Athens, Greece for the kind advices on his gradient theory, who stayed in the author's laboratory from Oct. 21, 2002 to Feb. 15, 2003.

\section{References}

1) Aifantis, E.C., On the microstructural origin certain inelastic models, J. Eng. Mater. Tech. (ASME), Vol.106, pp.326-330, 1984.

2) Aifantis, E.C., A proposal for continuum with microstructure. Mech. Res. Comm., Vol.5, pp.139-145, 1978.

3) Vardoulakis, I. and Aifantis, A.C., Gradient flow theory of plasticity for granular materials, Acta Mech., Vol.87, pp.197-217, 1991.

4) Vardoulakis, I. and Frantziskonis, G., Micro-structure in kinematic-hardening plasticity. Eur. J. Mech. A/Solids, Vol.11, No. 4, pp.467-486, 1992.

5) Vardoulakis, I. and Sulem. J., Bifurcation Analysis in Geomechanics, Blackie Academic \& Professional, London, 1995.

6) Drucker, D.C., Conventional and unconventional plastic response and representation. Appl. Mech. Rev. (ASME), Vol.41, pp.151-167, 1988.

7) Hashiguchi, K., Constitutive equations of elastoplastic materials with elastic-plastic transition, $J$. Appl. Mech. (ASME), Vol.47, pp.266-272, 1980.

8) Hashiguchi, K., Subloading surface model in unconventional plasticity, Int. J. Solids Struct., Vol.25, pp.917-945, 1989.

9) Hashiguchi, K. and Chen, Z.-P., Elastoplastic constitutive equations of soils with the subloading surface and the rotational hardening, Int. J. Numer. Anal. Meth. Geomech., Vol.22, pp.197-227, 1998.

10) Hashiguchi, K., Saitoh, K., Okayasu, T. and Tsutsumi, S., Evaluation of typical conventional and unconventional plasticity models for prediction of softening behavior of soils, Geotechnique, Vol.52, pp.561-573, 2002.

11) Hashiguchi, K., The tangential plasticity, Metals and Materials, Vol.4, pp.652-656, 1998.

12) Hashiguchi, K. and Tsutsumi, S., Elastoplastic constitutive equation with tangential stress rate effect. Int. J. Plasticity, Vol.17, pp.117-145, 2001.

13) Hashiguchi, K., Gradient elastoplastic constitutive equation extended to the subloading surface model with the tangential plasticity, Proc. 38th Annual Meeting of Japan Geotech. Soci., 1127-1128, 2003.

14) Hashiguchi, K., On the loading criterion, Int. J. 
Plasticity, Vol.10, pp.871-878, 1994.

15) Hashiguchi, K., Fundamentals in constitutive equation: continuity and smoothness conditions and loading criterion, Soils and Foundations, Vol.40, N.3, pp.155-161, 2000.

16) Hashiguchi, K., Fundamental requirements and formulation of elastoplastic constitutive equations with tangential plasticity. Int. J. Plasticity, Vol.9, pp.525-549, 1993.

17) Hashiguchi, K., Mechanical requirements and structures of cyclic plasticity models. Int. J. Plasticity, Vol.9, pp.721-748, 1993.

18) Hashiguchi, K., The extended flow rule in plasticity. Int. J. Plasticity, Vol.13, pp.37-58, 1997.

19) Rudnicki, J.W. and Rice, J.R., Conditions for localization of deformation in pressure-sensitive dilatant materials. J. Mech. Phys. Solids, Vol.23, pp.371-394, 1975.

20) Storen, S. and Rice, J.R., Localized necking in thin sheet. J. Mech. Phys. Solids, Vol.23, pp.421-441, 1975.

21) Vermeer, P., Upgrading of soil models by Hencky's theory of plasticity, Modern Approaches to Plasticity (ed. by D. Kolymbas), Elsevier, pp.71-82, 1993.
22) Papamichos, E., Vardoulakis, I. and Han, C., Noncoaxial flow theory of plasticity: shear failure prediction in sand, Modrern Approaches to Plasticity (ed. by D. Kolymbas), Elsevier pp.585-598, 1993.

23) Papamichos, E. and Vardoulakis, I., Shear band formation in sand according to non-coaxial plasticity model. Geotechnique, Vol.45, pp.649-661, 1995.

24) Yatomi, C., Yashima, A., Iizuka, A. and Sano, I., General theory of shear bands formation by a non-coaxial Cam-clay model. Soils and Foundations, Vol.29, No.3, pp.41-53, 1989.

25) Hashiguchi, K., Saidi, A.R. and Naghdabadi, R., Large deformation theory by the logarithmic strain tensor and the subloading surface model with tangential plasticity, Proc. 58th Annual Meeting of JSCE, submitted, 2003.

26) Naghdabadi, R. and Saidi, A.R., Kinematic and constitutive modeling of elastic-plastic hardening materials based on logarithmic strain, Proc. 6th Biennial Conf. Eng. Systems Design and Anal., ASME-ESDA2002, Istanbul., pp. 1-8, 2002.

(Received April 18,2003) 